\title{
Gut immune system and oral tolerance
}

\author{
Patricia Castro-Sánchez and José M. Martín-Villa* \\ Departamento de Inmunología, Facultad de Medicina, Pabellón V: Planta 4, Universidad Complutense de Madrid, 28040 \\ Madrid, Spain
}

\section{Abstract}

Gut mucosal surfaces separate the external environment from the internal sterile environment and so represent a first line of defence system. This barrier faces environments rich in pathogens that have developed effective mechanisms for colonisation of epithelial surfaces and invasion of mucosal tissues, but also harmless antigens such as food, airborne antigens or commensal bacterial flora. The latter represent the vast majority of the encountered antigens and require an appropriate response characterised by either ignorance or active suppression. However, for the former, a robust immune response is needed. Mucosae have developed a complex immune system that is capable of mounting an immune response against pathogenic antigens, while maintaining the required ignorance or active suppression against non-pathogenic antigens. Taking advantage of this knowledge, strategies have been devised to induce oral tolerance to antigens involved in experimental autoimmune disease or human conditions. It is now known that oral tolerance induces the up-regulation and activation of $\mathrm{T}$ cells with regulatory properties, a subtype of $\mathrm{CD}^{+}{ }^{+} \mathrm{T}$ cells whose function is to regulate functions of other $\mathrm{T}$ lymphocytes to avoid excessive immune activation. Amongst them, the Th3 cells (cells that express the latency-associated peptide on the surface and secrete transforming growth factor $\beta$, a cytokine with immunoregulatory properties) are especially relevant in the induction of oral tolerance. Orally fed antigens seek to generate these types of cells in the treatment of autoimmune diseases in experimental animals or human subjects.

\section{Key words: Mucosal immunity: Mucosa-associated lymphoid tissue: Regulatory T cells: Oral immunisation}

\section{Gut-associated lymphoid tissue}

The mucosal immune system is the part of the immune system juxtaposed to the mucosal surfaces and in direct contact with the external antigenic environment. It is composed of the lymphoid tissues that are associated with mucosal surfaces (mucosa-associated lymphoid tissue) and can be separated into several components: gut-associated lymphoid tissue (GALT), bronchus-associated lymphoid tissue (BALT), nasopharynx-associated lymphoid tissue (NALT), the mammary and salivary glands and the genitourinary organs.

Gastric mucosal tissues are heavily populated with cells of the immune system. It is estimated that the intestinal lining contains more lymphoid cells and produces more antibodies than any other organ in the body ${ }^{(1)}$. However, the immune cells are not alone in the task of preventing infection at mucosal surfaces. The gut epithelial barrier constitutes a key element in preventing penetration of micro-organisms. The cells that compose the intestinal mucosal barrier undergo continuous renewal from pluripotent stem cells located near the base of the crypts of Lieberkühn. Progeny of these stem cells differentiate into one of four cell lineages as they migrate towards the crypt surface (enterocyte, goblet cells or enteroendocrine cells) or to the crypt base (Paneth cells). Enterocytes are responsible for absorption of nutrients and secretion of electrolytes and also represent central components of the mucosal immune system ${ }^{(2)}$.

Intestinal epithelial cells form a highly selective barrier. Transcellular and paracellular fluxes are tightly controlled by membrane pumps, ion channels and tight junctions, adapting permeability to physiological needs. Besides this mechanical protective function, the epithelium provides the immune system with a continuous flux of information about the external environment. A correct function of the mucosal immune system requires transport of molecules and antigens through the epithelial barrier and the establishment of collaboration among the epithelial cell, the professional antigen-presenting cell and lymphoid cells. Tolerance against commensals and immunity against pathogens require intact antigen uptake recognition, processing and response mechanisms ${ }^{(3,4)}$.

Abbreviations: DC, dendritic cells; GALT, gut-associated lymphoid tissue; HLA, human leucocyte antigen; IEL, intraepithelial lymphocytes; IFN- $\gamma$, interferon $\gamma$; LAP, latency-associated peptide; M, microfold; MHC, major histocompatibility complex; MLN, mesenteric lymph nodes; PP, Peyer's patches; TGF- $\beta$, transforming growth factor; Treg, regulatory T cells.

*Corresponding author: J. M. Martín-Villa, fax +34 913941641, email jmmvilla@med.ucm.es 


\section{Structure of the gut-associated lymphoid tissue}

The lymphoid elements of different GALT can be morphologically and functionally subdivided into two major parts: the organised GALT, consisting of mucosal follicles that are responsible for the induction phase of the immune response, and the diffuse GALT, which consists of widespread leukocytes scattered throughout the epithelium and the lamina propria of the mucosa and constitute the effector sites ${ }^{(5,6)}$

\section{Organised gut-associated lymphoid tissue}

The organised GALT (mucosal inductive site) includes the ileal Peyer's patches (PP), the mesenteric lymph nodes (MLN) and the appendix, and can be used as a model for the other mucosa-associated components.

(1) Peyer's patches. The PP are macroscopic lymphoid aggregates that are found in the submucosa along the length of the small intestine.

Mature PP consist of collections of large B-cell follicles and intervening T-cell areas. B naïve cells form the germinal centre of the follicle, supported or connected by follicular dendritic cells (DC). These follicular DC are not bone marrow-derived and are different from the DC that present antigens to the naïve $\mathrm{T}$ cells. Each follicle is surrounded by a parafollicular area rich in T cells, where a large number of high endothelium venules exist, allowing cellular migration and lymphoid recirculation. PP differ from lymph nodes elsewhere in the body because they lack afferent lymphatics. This characteristic is in keeping with the notion that antigen is sampled from the lumen via the overlying epithelium.

The lymphoid areas are separated from the intestinal lumen by a single layer of columnar epithelial cells, known as the follicle-associated epithelium, and a more diffuse area immediately below the epithelium, known as the subepithelial dome. The follicle-associated epithelium differs from the epithelium that cover the mucosa, as it has lower levels of digestive enzymes, a less pronounced brush border and a total absence of receptor for polymeric IgA. It is infiltrated by large numbers of $\mathrm{B}$ cells, $\mathrm{T}$ cells, macrophages and DC. The most notable feature of the follicle-associated epithelium is the presence of microfold (M) cells. M cells are specialised enterocytes with poorly developed brush borders and a thin overlying glycocalyx, and thus are adapted to an antigen uptake function. $M$ cells differentiate from enterocytes under the influence of membrane-bound lymphotoxin- $\alpha_{1} \beta_{2}$ that is present on local lymphoid cells, mainly B cells ${ }^{(5,7)}$

(2) Mesenteric lymph nodes. MLN are the largest lymph nodes in the body. Their development is distinct from that of both PP and peripheral lymph nodes, as it is relatively unaffected by the absence of most of the factors that are involved in the ontogeny of these other organs such as TNF or TNF receptor, amongst others. These factors might have complementary roles in MLN development. Accumulation of lymphocytes in MLN also requires L-selectin and $\alpha_{4} \beta_{7}$ integrin adhesion molecules, which normally direct lymphocytes to enter peripheral and mucosal tissues, respectively. As a result of these unique anatomical features, the MLN might be a crossroad between the peripheral and mucosal recirculation pathways ${ }^{(7)}$.

In either case, the first step in the induction of a mucosal immune response is the transport of antigens across the epithelial barrier. Following antigen processing and presentation in inductive sites, IgA-committed antigen-specific B lymphoblasts proliferate locally and migrate via the bloodstream to local and distant mucosal and secretory tissues. There, they differentiate primarily into polymeric IgA-producing plasma cells. Dimeric or polymeric IgA antibodies are transported across the epithelial cells into glandular and mucosal secretions via receptor-mediated transcytosis ${ }^{(8)}$.

\section{Diffused mucosa associated lymphoid tissue}

The less organised lymphoid elements associated with the intestinal epithelium and the lamina propria contains both the lamina propria mononuclear cells and the intraepithelial lymphocytes (IEL).

(1) Lamina propria mononuclear cells. The lamina propria is the layer of connective tissue between the epithelium and the muscularis mucosa. This layer is made up of smooth muscle cells, fibroblasts, lymphatics and blood vessels ${ }^{(7)}$.

Adult human large and small intestinal lamina propria is infiltrated of lymphoid and myeloid cells. T cells, B cells, macrophages, DC, neutrophils, other granulocytes and mast cells are found. The large numbers of macrophages, DC and $\mathrm{T}$ cells in the lamina propria make it likely that antigens crossing the epithelium may be processed and presented to lamina propria $\mathrm{CD}^{+}{ }^{+} \mathrm{T}$ cells. Macrophages are one of the most abundant leucocytes in the intestine, present all over the intestinal tract of mammals and being especially abundant in the lamina propria. These cells are highly phagocytic and have strong bactericidal activity and, yet, they produce anti-inflammatory cytokines such as IL-10. This is of considerable importance as intestinal macropahges can thus ingest microbes without necessarily producing a local inflammatory response. Furthermore, this population plays an important role in regulating antigen-specific tolerance and the development of antigenspecific $\mathrm{CD}^{+}$regulatory T cells (Treg) (given later), a crucial step to prevent inflammatory reactions against commensal bacteria and food proteins ${ }^{(9)}$.

IgA plasma cells make up 30-40\% of the mononuclear cells in human intestinal lamina propria and small B cells make up $15-45 \%$ of the cells. In the normal intestine, about $80 \%$ of the total plasma cells secrete IgA.

$\mathrm{T}$ cells in the lamina propria are mainly $\mathrm{CD}^{+}(60-70 \%)$ and the vast majority express a T-cell receptor composed by the $\alpha$ and $\beta$ chains (TcR $\alpha \beta)(95 \%)$. About $10 \%$ of them are $\mathrm{CD} 25^{+}$, and most express CD45RO, indicating a memory phenotype. $\mathrm{CD}^{+}{ }^{+} \mathrm{T}$ cells in the lamina propria are of particular importance to local immune regulation. They are generally unresponsive to TcR-mediated proliferative signals, but in human subjects, they can be induced to proliferate when CD2 is used as an accessory molecule. They produce large amounts of cytokines, particularly interferon $\gamma$ (IFN- $\gamma$ ), but also IL-4 and IL-10. Lamina propria $\mathrm{CD}^{+} \mathrm{T}$ cells can also 
have potent cytolytic T-lymphocyte activity. Some of the antigen-experienced lamina propria $\mathrm{T}$ cells might be true effector cells and might help local B cells to produce IgA. Alternatively, they can be effector memory cells. Finally, lamina propria $\mathrm{T}$ cells might be Treg and therefore responsible for maintaining local tolerance to environmental antigens (given later)

(2) Intraepithelial lymphocytes ${ }^{(10,11)}$. The average number of IEL in adult human jejunum is twenty per 100 absorptive cells and decreases distally in the gut. These cells are located above the basal lamina in the epithelial layer and separated from adjacent enterocytes by a $10-20 \mathrm{~nm}$ space. These lymphocyte-epithelial cell contacts have no junctional structure. Intraepithelial cells are a functionally heterogeneous population that contains cells with antitumor activity, natural killer activity, allospecific cytotoxic T lymphocytes, precursors of cytotoxic $\mathrm{T}$ lymphocytes and mast cells. Almost all IEL are $\mathrm{CD}^{+}$. Among these cells, only 5-15\% express CD4 and the remaining cells express CD8. In the intestinal epithelium, the proportion of $\gamma \delta^{+}$lymphocytes is much larger than in the peripheral blood and lamina propria, and thus IEL are thought to have a special role. The microenvironment within the intestinal epithelium may influence the differentiation of IEL. Although the functions of IEL are unclear, some possibilities are cytotoxicity, cytokine secretions, regulation of renewal of mucosal epithelium and tolerance.

Almost all IEL, whatever their surface phenotype, bear the $\alpha_{\mathrm{E}} \beta_{7}$ integrin (CD103), whose ligand is epithelial E-cadherin. Interactions between $\alpha_{\mathrm{E}} \beta_{7}$ and E-cadherin may help anchor IEL in the epithelium and may also play a functional role ${ }^{(12)}$.

Extrathymic differentiation of $\mathrm{IEL}^{(13)}$ is not incompatible with the passage through the thymus of some $\mathrm{TcR}^{-}$progenitor cells, which my undergo $\mathrm{T}$ lineage commitment and or expansion in this location. The release of cytokines, such as IL-7, by the thymus or by antigenically stimulated thymusderived cells may also influence the development of thymusindependent IEL $^{(14)}$. IL-7, necessary for the earliest thymic lymphocyte precursors, has been detected abundantly in fetal intestinal epithelial cells and CD117 (stem cell factor receptor) on occasional cells in the lamina propria. These findings, with the evidence that maturation and TcR recombination occur in situ in fetal intestine, support the idea of extrathymic T-cell differentiation in the gut ${ }^{(15)}$.

\section{Induction of the immune response}

There is abundant evidence that organised mucosa-associated lymphoid tissue plays a major role in antigen sampling and generation of lymphocytes, including specific IgA effector $\mathrm{B}$ cells, memory $\mathrm{B}$ cells and $\mathrm{T}$ cells. This involves active lymphocyte proliferation, local production of certain cytokines and continuous cellular trafficking. Antigen uptake and initiation of an immune response is tightly regulated because an adequate immune response requires first access to antigen. These structures lack lymphatic supply of antigens, but instead can sample foreign material from epithelial surfaces $^{(12)}$.

\section{Initial mucosal antigen encounter/antigen uptake}

Antigen uptake is the initial step required for the induction of the adequate immune response. This uptake may be carried out by three different pathways: specialised $M$ cells, DC and epithelial cells (enterocytes).

(1) $M$ cells. In simple intestinal epithelia, whose intercellular spaces are sealed by tight junctions, antigens are preferentially taken up through the specialised areas of the follicle-associated epithelium. Specialised epithelial $M$ cells deliver samples of foreign material by transepithelial transport from the lumen to the organised lymphoid tissues within the mucosa ${ }^{(16,17)}$. M cells, through its limited microvillus border, sparse glycocalyx and active transcytotic pathway, are clearly important in delivering certain types of antigens to PP for both initiating immune responses, characterised by active IgA secretion, and contributing to the development of oral tolerance ${ }^{(18)}$.

The M cell basolateral surface is deeply invaginated to form a large intraepithelial pocket into which transcytosed particles and macromolecules are delivered. Antigens are efficiently endocytosed or phagocytosed by multiple mechanisms in the apical membrane of the M cells. Each of these mechanisms results in antigen transport into endosomal tubules and vesicles and large multivesicular bodies, and to their subsequent release by exocytosis into the pocket. It is not known whether $\mathrm{M}$ cells participate in the processing and presentation of antigens, nor if they express major histocompatibility complex (MHC) class II molecules. Instead they are believed to pass on intact antigens to professional antigen presenting cells (APC) either in the epithelium or in the underlying dome region.

Specific subpopulations of lymphocytes migrate into the pocket and associate closely with the pocket membrane, forming apparent adhesion sites. In rodents, rabbits and human subjects, B and T lymphocytes, with a small number of macrophages, have been identified. Most of the T cells are $\mathrm{CD}^{+}$, none were TcR $\gamma \delta^{+}$and most of them express the early activation marker CD69, typical of memory cells, although in some species naïve T cells are observed. B cells in the pockets express the naïve cell marker CD45RA, along with human leucocyte antigen (HLA)-DR, suggesting that the M cell pocket is a site of interaction of $\mathrm{T}$ cells with antigen-presenting $\mathrm{B}$ cells. This interaction might lead to IL-2 secretion and promote T-cell survival and proliferation or anergy and hence tolerance, should this naïve T cell lack the necessary co-stimulatory molecules ${ }^{(12)}$

$\mathrm{B}$ cells in the $\mathrm{M}$ pockets are of the same type as the subepithelial B cells associated with the underlying follicle. It has been suggested that $B$ lymphoblast traffic into the $M$ cell pocket may allow continued antigen exposure and extension and diversification of the immune response. The cells in the pocket may interact early with the incoming antigens, in an environment sequestered from the modulating influence of systemic humoral immunity.

Below the epithelium of the dome lies an extensive network of macrophages and DC intermingled with $\mathrm{CD}^{+}$ $\mathrm{T}$ cells and $\mathrm{B}$ cells from the underlying follicle. These cell populations are presumably active in uptake of incoming 
pathogens as well as in processing, presentation and perhaps storage of antigens. Several DC subsets have been described in mouse $\mathrm{PP}^{(19)}$.

From there, antigen-presenting cells move to T-cell areas or B-cell follicles, where they can interact with naïve lymphocytes. In the follicle, B cells undergo immunoglobulin class switching, from expression of IgM to IgA, under the influence of several local factors, including transforming growth factor (TGF- $\beta$ ), IL-10 and cellular signals delivered by DC and $\mathrm{T}$ cells.

The lymphocytes primed in the PP exit through the draining lymphatics to the MLN, where they reside for an undefined period for further differentiation before they migrate into the bloodstream through the thoracic duct and finally accumulate in the mucosa. Lymphocytes primed in the GALT lose expression of L-selectin and selectively up-regulate the expression of $\alpha_{4} \beta_{7}$ integrin, whose ligand, mucosal addressin cell adhesion molecule 1 , is expressed at high levels by the vasculature of mucosal surfaces ${ }^{(7,20)}$.

(2) Dendritic cells. Antigen-presenting cells such as macrophages and DC are positioned immediately below $\mathrm{M}$ cells and thus are ideally located to sample transported antigens that are subsequently presented to lymphoid follicles. These sites are relatively rare within the epithelial surface, and they alone may not be sufficient to representatively sample the vast lumenal content ${ }^{(21)}$.

It has been reported that the adaptive immune system appears to display an additional, M-cell independent, mechanism that allows DC to sample environmental micro-organisms without compromising the epithelial barrier function and to deliver them into lymphoid tissues where an efficient immune response can be mounted. DC extend processes through epithelial tight junctions into the lumen and are able to sample lumenal content ${ }^{(20)}$. The DC processes slide between intestinal epithelial cells, preserving the epithelial cell barrier making effective tight junctions with intestinal epithelial cells, as DC are shown to express tight junction proteins themselves and no detectable alteration in tight junction permeability is found. Under resting conditions, infiltrating DC would establish loose contacts with pre-existing epithelial tight junctions. Upon bacterial infection, DC would be recruited from the blood and activated, probably via epithelial signals. They then would up-regulate the expression of tight junction proteins and distribute them to the cell surface and dendrites. This would allow DC to compete for epithelial proteins and open up the tight junction. Infiltrating DC then would face the gut lumen and could directly sample bacteria. Bacterial components, such as lipopolysaccharides, trigger reorganisation of tight junction proteins, allowing DC to detach from the junctions with epithelial cells and to migrate into the draining lymph nodes, after a change in the chemokine receptor programme also triggered by bacterial components.

Because DC are migratory cells, they can transport pathogens to the MLN and the spleen for the induction of systemic responses, which suggests that this alternative route of bacterial internalisation has an important physiological relevance. It suggests the implication of DC in direct IgA induction against commensal bacteria in the lamina propria by synthesising TGF- $\beta$, a cytokine involved in IgA switch, and could also be involved in the transport of apoptotic intestinal epithelial cells to T-cell areas of MLN without perturbing the integrity of the epithelial barrier ${ }^{(19)}$.

(3) Epithelial cells (enterocytes). Enterocytes are relevant components of the gut immune response ${ }^{(22)}$. These cells are able to sense pathogens through Toll-like receptors or Nodlike receptors ${ }^{(23)}$ and express molecules involved in antigen presentation (MHC-I, MHC-II) and T-cell binding and potentially co-stimulation ${ }^{(24-26)}$. In addition to expressing antigenpresenting molecules and the machinery necessary for antigen presentation functions, intestinal epithelial cells express (IL-8 ${ }^{(24)}$ ) and respond to (IL-21R ${ }^{(27)}$ ) a wide variety of cytokines. Regulated production of a repertoire of inducible/ inflammatory and constitutive/homeostatic chemokines by the intestinal epithelium orchestrates and coordinates trafficking of mucosal inflammatory and immune effector cells. Enterocyte-derived chemokines likely play a major role in regulating leucocyte migration in chronic deregulated inflammation, characteristic of inflammatory bowel disease and during infection with microbial pathogens ${ }^{(28)}$.

The vast majority of the mucosal surface is comprised of the absorptive intestinal epithelium, which is predicted to be the major site of antigen contact. The availability of antigens via this cell barrier is regulated by soluble mediators, including cytokines and toxins that increase permeability (IL-4, IL-13, TNF and IFN- $\gamma$ ) and cytokines that enhance the barrier formation (TGF- $\beta$ and IL-15). Paracellular permeability is an indiscriminate mechanism of antigen uptake that delivers antigens directly to the basolateral surface of the intestinal epithelial cell and to professional antigen-presenting cells such as macrophages and DC. More important are the transcellular mechanisms, non-receptor mediated (fluid phase) or receptor mediated. The former is predominantly degradative, with a minor fraction traversing the cell intact, although given the large surface area, this pathway may be biologically relevant. Receptor-mediated mechanisms serve to transport intact molecules. Both processes have the capability of delivering macromolecules into endolysosomal compartments associated with MHC class II antigen presentation in epithelial cells ${ }^{(18)}$.

Numerous reports have described a low expression of HLA class II antigens on the surface of normal epithelial cells, and have demonstrated increased expression of these molecules associated with pathological conditions such as inflammatory bowel disease, graft $v$. host disease and celiac disease. These observations suggested that the intestinal epithelium might function in the immigration or regulation of $\mathrm{CD}^{+} \mathrm{T}$ cell response in the mucosa.

Several groups have described the presentation of antigens by intestinal epithelial cells in human or animal models ${ }^{(29)}$. Two distinct pathways for antigen processing by intestinal epithelial cells that distinguish between the activated and non-activated states have been described. The first pathway is similar to that seen in conventional antigen-presenting cells and uses similar proteases, invariant chain and human leucocyte antigen (HLA)-DM. This pathway facilitates efficient antigen presentation, even at low antigen concentrations, and 
occurs in the presence of the proinflammatory cytokine IFN- $\gamma$. The second pathway functions independent of invariant chain and HLA-DM $\alpha \beta$, occurs in the absence of IFN- $\gamma$ and requires high concentration of antigen to elicit T-cell stimulation. This non-conventional pathway seems associated with oral tolerance.

Human intestinal epithelial cells express classical MHC class I, as well as a number of non-classical MHC molecules: MHC class I-related chain molecules A and B (MICA and MICB), human leucocyte antigen-E (HLA-E), hereditary hemochromatosis gene (High Ferrum, HFE) product, cluster of differentiation antigen $1 \mathrm{~d}$ (CD1d) and the neonatal $\mathrm{Fc}$ receptor (FCRn). The in vivo role of classical MHC class I is presumably related to immunosurveillance of intracellular infections, given the presence of MHC class I-restricted, antigen-specific cytolytic T-cell effector cells within the epithelium. A similar function is likely attributable to MICA and MICB. CD1d expression on epithelium has been demonstrated in rodents and human subjects. It functions in the presentation of lipid antigens to NK-T cells $\left(\mathrm{CD}^{+}{ }^{+}\right.$and double-negative $\mathrm{T}$ cells bearing an invariant T-cell receptor $\alpha$ chain (V $\alpha 24)$ in association with V $\beta 11$ and expressing the natural killer cell marker NKRP1A in vitro). If such response occurs in vivo, CD1d might function in the acquisition of lipid antigens apically and presentation basally to local $\mathrm{T}$ cells homologous to the hypothetical role of the MHC class II pathway for presentation of intraluminal protein antigens.

\section{Oral tolerance}

Immunological tolerance is a mechanism by which one of the crucial characteristics of the immune system (lack of reactivity against self) is achieved. Elements of the immune system should not initiate a harmful response against own constituents, and several mechanisms have been proposed to achieve this goal. The classical view of tolerance mechanisms, relying solely on neonatal deletional events, is no longer dominant, and it is assumed now that it requires an active process that takes place throughout the entire life of the individual ${ }^{(30)}$. In fact, it is considered that it involves the activity of noninflammatory/regulatory auto-reactive $\mathrm{T}$ lymphocytes present at a stable frequency in the normal repertoire ${ }^{(31)}$

As constituents of the immune system, elements of GALT also fulfil this condition and preserve self-antigens. However, and inherent to its surveillance of a mucosal surface, GALT (and also members of mucosa-associated lymphoid tissue) must, on the one hand, tolerize foreign antigens that enter the body through the digestive tract, but that, nevertheless, are beneficial and, on the other hand, detect and react to potential harmful antigens entering through the same route.

Oral tolerance, in this sense, is of unique immunologic importance as it is a continuous natural immunological event driven by exogenous antigen ${ }^{(30)}$. Most of the encounters with antigens take place at the mucosal surfaces. Given the large area of the gut (as mentioned earlier), there is a continuous antigenic stimulation that results mostly in the induction of oral tolerance rather than inflammatory immune reactions. The main role of this 'physiological' oral tolerance is to accept as self-components the foreign antigens that enter the body through the gut. Disruptions of this process may end up in pathological conditions.

Induced oral tolerance takes advantage of the properties of GALT and manipulates it in such a way that specific suppression of cellular or humoral immune responses to antigens is achieved by prior administration of the antigen by the oral route $^{(32)}$. The idea underlying this procedure is that once an individual is orally treated with an antigen, no response is mounted against this antigen on subsequent encounters via its natural route. This manipulation of the immune system may be adequate to treat pathologies with an underlying autoimmune or auto inflammatory reaction (autoimmune diseases, colitis, stroke, etc.)

\section{Mechanisms of oral tolerance}

The main mechanisms of induction of oral tolerance are mediated by Treg, known to mediate suppression reactions. Treg are a subtype of $\mathrm{CD}^{+}{ }^{+} \mathrm{T}$ cells, scarcely representing $5 \%$ of all $\mathrm{CD}^{+}{ }^{+}$cells. Their major role is to regulate functions of other $\mathrm{T}$ lymphocytes to avoid excessive immune activation and thus autoimmune disease or autoinflammatory reactions. Treg actually comprises several subsets of phenotypically similar cells that mediate suppression by various cells ${ }^{(33)}$. Though this regulatory function is mainly circumscribed to $\mathrm{T}$ cells of the $\mathrm{CD}^{+}$phenotype, $\mathrm{CD}^{+} \mathrm{T}$ cells with regulatory functions have been recently described ${ }^{(34,35)}$.

It is known that orally fed antigens are able to induce all kinds of $\mathrm{T}$ cells with regulatory properties (see Table 1) and even Treg cells of the CD8 phenotype. Amongst them, especially relevant is the activation of Th3 Treg cells. These cells produce TGF- $\beta$ (a cytokine with known regulatory properties) and IFN- $\gamma$, exert a regulatory function and express latency-associated peptide (LAP) on their surface. This cell subtype was described by Weiner et $a l .^{(36)}$ and their role in oral immunoregulation widely studied by this group.

\section{Transforming growth factor $\beta$ and latency-associated peptide}

TGF- $\beta$ is a member of the TGF- $\beta$ superfamily, which includes more than thirty structurally related proteins. This superfamily includes TGF- $\beta$, activins, bone morphogenetic proteins and growth and differentiation factors.

The TGF- $\beta$ family itself includes three different types (TGF- $\beta$ 1-3) in mammals and their main biological activities include regulation of cell proliferation, control of extracellular matrix components expression and inhibition of the immune system. Mature TGF- $\beta$ are $25 \mathrm{kDa}$ proteins of two

Table 1. Induction of distinct regulatory $\mathrm{T}$ cells (Treg) upon oral immunisation

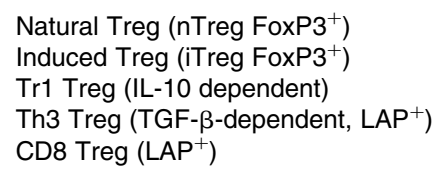

TGF- $\beta$, transforming growth factor; LAP, latency-associated peptide. 
polypeptides of $12.5 \mathrm{kDa}$, derived from a $55 \mathrm{kDa}$ protein that dimerises after synthesis. Once dimerised, the protein is proteolytically processed by furin-like proteases to yield a C-terminal end, which corresponds to the mature growth factor, and the N-terminal end, which is referred to as the LAP. LAP remains non-covalently bound to TGF- $\beta$ and this peptide refrains the TGF- $\beta$ from exerting its function (hence the name latency), and needs to be removed for the mature factor to achieve its full activity, a process called activation of TGF- $\beta$ (see Fig. 1). This activation can be achieved by various ways, either in vitro (using chaotropic agents) or in vivo (by integrins, thrombospondin or proteinases) ${ }^{(37)}$.

\section{Latency-associated peptide and T lymphocytes: latency- associated peptide expression on regulatory $T$ cells}

As mentioned earlier, TGF- $\beta$ is an immunoregulatory cytokine that controls immune responses ${ }^{(38)}$. It is known that TGF- $\beta$ is able to induce Th17 differentiation ${ }^{(39,40)}$ and plays a role in autoimmunity: TGF- $\beta$-deficient mice manifest autoimmune diseases.

Many cell types are able to produce TGF- $\beta$, but it is the T-cell-produced TGF- $\beta$, the one that seems to play a pivotal role in the control of autoimmune responses and Th17 differentiation. T-cell specific TGF- $\beta$ conditional knockout develop autoimmune diseases and fail to generate Th17 cells ${ }^{(41)}$. It is not fully known how this T-cell-produced TGF- $\beta$ is activated.

The expression of the LAP-TGF- $\beta$ complex on the surface of regulatory Treg was initially described by Nakamura on murine $\mathrm{CD}^{+} \mathrm{CD}^{+} 5^{+}$cells $^{(42)}$ and later reported on Foxp3 ${ }^{+}$ $\mathrm{T}$ cells ${ }^{(43)}$, Foxp3 ${ }^{-} \mathrm{T}$ cells and $\mathrm{CD}^{+}$cells, all of them able to exert a regulatory function. It is not clear how this function is carried out, but it is possible that surface LAP/TGF- $\beta$ on $\mathrm{T}$ cells can trigger TGF- $\beta$ signalling in target cells by a cell-cell contact manner. Membrane-bound TGF- $\beta$ is an important component of certain Treg populations and may or may not be expressed on all FoxP3 ${ }^{+}$Treg.

DC in the gut are key cells in the generation and regulation of intestinal immune responses. These cells express Toll-like receptors and Nod-like receptors and are actively involved in the uptake of antigens and in the subsequent events taking place. Once the DC is activated, it migrates to the nearby lymph node and present antigens to naïve $\mathrm{T}$ or $\mathrm{B}$ cells. In this setting, T-cell differentiation into Th1 (secreting IL-12), Th2 (IL-4) or regulatory Th3 or Tr1 cells (producing IL-10 or TGF- $\beta$ ), and thus the fate of the immune response (tolerization or rejection), depends on the receptor engaged in the DC activation ${ }^{(23)}$. The induction of regulatory cells in the gut milieu is thus related to gut DC that have special properties, which result in the preferential induction of Treg.

\section{Induction of oral tolerance}

The induction of oral tolerance to antigens has been intensively investigated in recent years in several animal experimental autoimmune diseases and human conditions ${ }^{(36)}$. The rational underlying this approach is the induction of adequate Treg cells to down-regulate the immune response against the target antigen.

\section{Choice of target antigen and bystander suppression}

Since the main goal of oral tolerance is inducing lack of specific response against a given antigen, it is relevant the

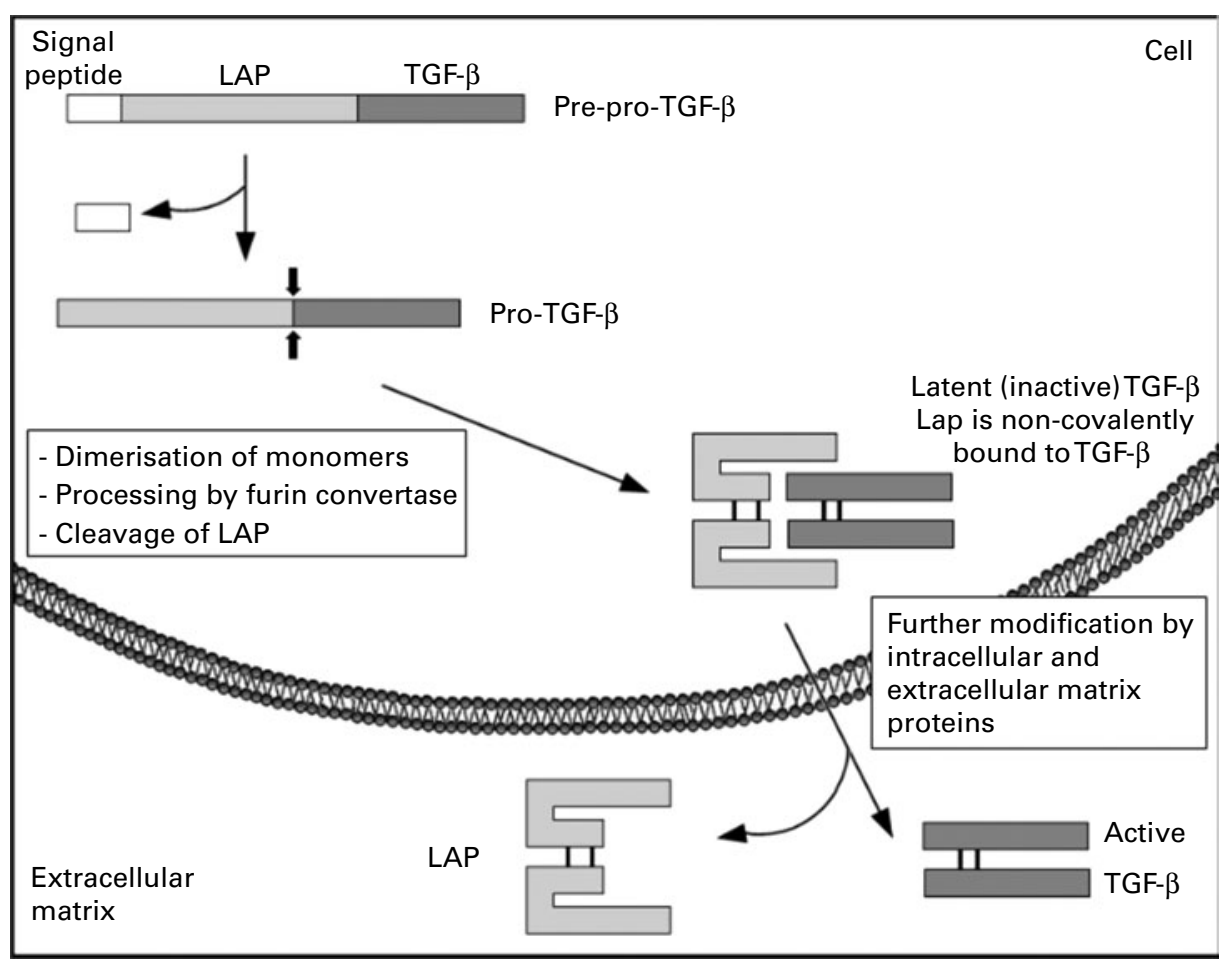

Fig. 1. Schematic representation of transforming growth factor $\beta$ (TGF- $\beta$ ) biosynthesis and activation. LAP, latency-associated peptide. 
choice of the antigen used for this procedure. However, it may not always be clear as to which is the major autoantigen in a given autoimmune disease. However, a mechanism known as bystander expression may help circumvent this problem. Bystander suppression $^{(44)}$ is a mechanism whereby regulatory cells induced by a fed antigen can induce tolerance to a different antigen, as long as both antigens (the fed and the target antigen) stand in close vicinity. This is obviously helpful in situations in which the autoantigen is not known or when there are multiple autoantigens in the tissue. In multiple sclerosis, there are three different potential autoantigens: myelin basic protein, proteolipid protein and myelin oligodendrocyte glycoprotein ${ }^{(45)}$.

The mechanism whereby this multiple suppression is achieved is based on the fact that Treg induced by the fed antigen secrete non-specific cytokines that then will suppress inflammation in the environment where the fed antigen is found. This means that for a given organ-specific inflammatory disease, it may not be required to know the specific target antigen, but just to feed an antigen able to migrate to the corresponding anatomic endroit, enabling tolerogenic responses to antigens nearby.

\section{Use of adjuvants in mucosal-induced immunological tolerance}

To improve the effect on immunosuppression of orally administered antigens, conjugation to adjuvants seems to be a fruitful approach to achieve this goal. Two of such adjuvants have yielded promising results: non-toxic B subunit protein of cholera toxin and its close analogue, the B subunit protein of Escherichia coli heat-labile enterotoxin). Conjugation to these molecules greatly diminished the concentration of antigen and the number of doses required to induce oral tolerization: even a single oral dose of antigen conjugate was effective ${ }^{(46,47)}$. This approach has been used in several models of autoimmune diseases or type I allergies and even in phase I/phase II human clinical trials in patients with Behcet's disease $^{(46)}$.

The mechanisms underlying the observed effects involve the generation of antigen-specific regulatory $\mathrm{CD}^{+}{ }^{+} \mathrm{T}$ cells (either $\mathrm{FoxP}^{+}$or $\mathrm{FoxP}^{-}$) that can suppress effector $\mathrm{T}$ cells and induce apoptosis and deletion of such cells, increase IL-10 or TGF- $\beta$ production (cytokines with immunoregulatroy properties) and induce tolerogenic $\mathrm{B}$ cells (given the ability of $\mathrm{B}$ cells to uptake cholera toxin B subunit (CTB)-coupled antigens via GM1 ganglioside receptors, increasing antigen uptake and antibody production by more than $10 \cdot 000$-fold $\left.{ }^{(48)}\right)$.

Since the induction of oral tolerance tends to shift the Th profile responses in the gut, it is then conceivable that the use of agents (such as cytokines) is able to modulate the immune system and drive it to a tolerance state. Administering such substances is a means to achieve the desired tolerization, and successful experiments have been published: intraperitoneal administration of IL-4, oral administration of IL-10 and IL- 4 or oral administration of LPS enhances oral tolerance to several antigens ${ }^{(49,50)}$.

\section{Tolerance induced by oral administration of CD3 monoclonal antibody}

Parenteral administration of CD3-specific monoclonal antibody has been long used as a therapeutic tool in several animal models of autoimmune diseases such as diabetes ${ }^{(51)}$ or allergic encephalitis ${ }^{(39)}$ and human trials have also been carried out. In addition, this antibody has been used as support therapy to control graft rejection in organ transplantation. It was then thought by Weiner and colleagues, of administering it orally in the setting of autoimmune diseases ${ }^{(52)}$.

Orally administered anti-CD3 antibody appeared in the villous epithelium within $30 \mathrm{~min}$ and increased 1 and $3 \mathrm{~h}$ after feeding. Further and contrary to intravenous supply antibody, orally fed anti-CD3 did not induce any TcR down-modulation, depletion of T cells, nor induce T-cell division and, very importantly, did not induce cytokine release syndromes (cytokine storm) or antiglobulin response. Its way of action is mediated by the induction of $\mathrm{CD}^{+}{ }^{+} \mathrm{CD} 25^{-} \mathrm{LAP}^{+}$-expressing $\mathrm{T}$ cells.

This approach has been tested in animal models ${ }^{(52)}$ and proved to be useful at preventing and treating established experimental autoimmune encephalitis or preventing autoimmune diabetes. In some instances, the $\mathrm{Fc}_{\mathrm{c}}$ portion of the antibody was not required, as orally fed $\mathrm{F}\left(\mathrm{ab}^{\prime}\right)_{2}$ fragments also achieved suppression.

This therapy opens new perspectives in the treatment of human pathologies, and on-going studies in human subjects have been reported for diabetes and non-alcoholic steatohepatitis ${ }^{(36)}$.

\section{Alternative routes: nasal immunisation}

On some occasions, induction of oral tolerance requires the use of large doses of antigen, which makes it difficult to achieve satisfactory results. Immunisation through other mucosal routes (such as nasal) then appears as an alternative methodology.

The nasal and upper respiratory tracts also present a mucosal immune system with two main structures, the nasal-associated lymphoid tissue and the bronchus-associated lymphoid tissue, which makes them suitable to achieve tolerance to antigens. One advantage of the respiratory immune system over the mucosal is the absence of digestion in the first, which is why small antigen amounts are able to drive the system to a state of tolerance, when compared with gut immunisation. The nasal antigen administration activates IL-10-producing DC (a special feature of the nasal-associated lymphoid tissue and bronchus-associated lymphoid tissue immune system, in contrast to TGF- $\beta$-producing gut DC), which are then able to activate $\operatorname{Tr} 1$ (IL-10 secreting) T cells.

This methodology has been assayed in a wide variety of experimental autoimmune diseases, such as experimental autoimmune uveitis, experimental autoimmune encephalitis or myasthenia gravis, diabetes or collagen-induced arthritis ${ }^{(53)}$.

\section{Conclusions/future perspectives}

The knowledge gained in recent years in the structure and function of the GALT and on different types of Treg, has 
shed light on the mechanisms of oral tolerance. This, in turn, has opened new possibilities for the treatment of autoimmune or inflammatory diseases. Future research will aim at devising therapies exploiting the potential of oral tolerance in the treatment of these diseases.

\section{Acknowledgements}

P. C.-S. planned and carried out the bibliography search and helped with the editing and discussion of the different drafts of the manuscript. J. M. M.-V. designed the content of the manuscript, planned the bibliography search and wrote the several drafts of the manuscript. Both authors read and approved the final version. There are no conflicts of interest to report. This manuscript was, in part, funded by a FIS grant PS09/02096.

\section{References}

1. McGhee JR, Mestecky J, Dertzbaugh MT, et al. (1992) The mucosal immune system: from fundamental concepts to vaccine development. Vaccine 10, 75-88.

2. Ponda PP \& Mayer L (2005) Mucosal epithelium in health and disease. Curr Mol Med 5, 549-556.

3. Baumgart DC \& Dignass AU (2002) Intestinal barrier function. Curr Opin Clin Nutr Metab Care 5, 685-694.

4. Moens E \& Veldhoen M (2012) Epithelial barrier biology: good fences make good neighbours. Immunology 135, 1-8.

5. Mowat AM, Millington OR \& Chirdo FG (2004) Anatomical and cellular basis of immunity and tolerance in the intestine. J Pediatr Gastroenterol Nutr 39, Suppl. 3, S723-S724.

6. Suzuki K, Kawamoto S, Maruya M, et al. (2010) GALT: organization and dynamics leading to IgA synthesis. Adv Immunol 107, 153-185.

7. Mowat AM (2003) Anatomical basis of tolerance and immunity to intestinal antigens. Nat Rev Immunol 3, 331-341.

8. Johansen FE \& Kaetzel CS (2011) Regulation of the polymeric immunoglobulin receptor and IgA transport: new advances in environmental factors that stimulate pIgR expression and its role in mucosal immunity. Mucosal Immunol 4, 598-602.

9. Mowat AM \& Bain CC (2011) Mucosal macrophages in intestinal homeostasis and inflammation. J Innate Immun 3, 550-564.

10. Cheroutre H, Lambolez F \& Mucida D (2011) The light and dark sides of intestinal intraepithelial lymphocytes. Nat Rev Immunol 11, 445-456.

11. Leon F (2011) Flow cytometry of intestinal intraepithelial lymphocytes in celiac disease. J Immunol Methods $\mathbf{3 6 3}$, 177-186.

12. Brandtzaeg P (2009) Mucosal immunity: induction, dissemination, and effector functions. Scand J Immunol $\mathbf{7 0}$, 505-515.

13. Rocha B (2007) The extrathymic T-cell differentiation in the murine gut. Immunol Rev 215, 166-177.

14. Rocha B, Guy-Grand D \& Vassalli P (1995) Extrathymic T cell differentiation. Curr Opin Immunol 7, 235-242.

15. Howie D, Spencer J, DeLord D, et al. (1998) Extrathymic T cell differentiation in the human intestine early in life. J Immunol 161, 5862-5872.

16. Pickard JM \& Chervonsky AV (2010) Sampling of the intestinal microbiota by epithelial M cells. Curr Gastroenterol Rep 12, 331-339.
17. Yamamoto M, Pascual DW \& Kiyono H (2012) M celltargeted mucosal vaccine strategies. Curr Top Microbiol Immunol 354, 39-52.

18. Blumberg RS, Lencer WI, Zhu X, et al. (1999) Antigen presentation by intestinal epithelial cells. Immunol Lett 69, $7-11$.

19. Rescigno M (2010) Intestinal dendritic cells. Adv Immunol 107, 109-138.

20. Rescigno M, Urbano M, Valzasina B, et al. (2001) Dendritic cells express tight junction proteins and penetrate gut epithelial monolayers to sample bacteria. Nat Immunol $\mathbf{2}$, 361-367.

21. Gewirtz AT \& Madara JL (2001) Periscope, up! Monitoring microbes in the intestine. Nat Immunol 2, 288-290.

22. Pabst R \& Rothkotter HJ (2006) Structure and function of the gut mucosal immune system. Adv Exp Med Biol 579, 1-14.

23. Sanz Y \& De Palma G (2009) Gut microbiota and probiotics in modulation of epithelium and gut-associated lymphoid tissue function. Int Rev Immunol 28, 397-413.

24. Rodriguez-Juan C, Perez-Blas M, Suarez-Garcia E, et al. (2000) Lens culinaris, Phaseolus vulgaris and vicia faba lectins specifically trigger IL- 8 production by the human colon carcinoma cell line CACO-2. Cytokine 12, 1284-1287.

25. Rodriguez-Juan C, Perez-Blas M, Valeri AP, et al. (2001) Cell surface phenotype and cytokine secretion in Caco-2 cell cultures: increased RANTES production and IL-2 transcription upon stimulation with IL-1beta. Tissue Cell 33, 570-579.

26. Martin-Villa JM, Ferre-Lopez S, Lopez-Suarez JC, et al. (1997) Cell surface phenotype and ultramicroscopic analysis of purified human enterocytes: a possible antigen-presenting cell in the intestine. Tissue Antigens 50, 586-592.

27. Abadie V, Sollid LM, Barreiro LB, et al. (2011) Integration of genetic and immunological insights into a model of celiac disease pathogenesis. Annu Rev Immunol 29, 493-525.

28. Dwinell MB, Johanesen PA \& Smith JM (2003) Immunobiology of epithelial chemokines in the intestinal mucosa. Surgery 133, 601-607.

29. Miron N \& Cristea V (2012) Enterocytes: active cells in tolerance to food and microbial antigens in the gut. Clin Exp Immunol 167, 405-412.

30. Faria AM \& Weiner HL (2006) Oral tolerance and TGF-betaproducing cells. Inflamm Allergy Drug Targets 5, 179-190.

31. Sakaguchi S (2004) Naturally arising $\mathrm{CD}^{+}{ }^{+}$regulatory $\mathrm{T}$ cells for immunologic self-tolerance and negative control of immune responses. Annu Rev Immunol 22, 531-562.

32. Faria AM \& Weiner HL (2006) Oral tolerance: therapeutic implications for autoimmune diseases. Clin Dev Immunol 13, $143-157$.

33. Whiteside TL (2012) Disarming suppressor cells to improve immunotherapy. Cancer Immunol Immunother $6 \mathbf{1}$, 283-288.

34. Zhang L, Bertucci AM, Ramsey-Goldman R, et al. (2009) Regulatory $\mathrm{T}$ cell (Treg) subsets return in patients with refractory lupus following stem cell transplantation, and TGF-beta-producing $\mathrm{CD}^{+}$Treg cells are associated with immunological remission of lupus. $J$ Immunol 183, 6346-6358.

35. Chen ML, Yan BS, Kozoriz D, et al. (2009) Novel CD8 ${ }^{+}$Treg suppress EAE by TGF-beta- and IFN-gamma-dependent mechanisms. Eur J Immunol 39, 3423-3435.

36. Weiner HL, da Cunha AP, Quintana F, et al. (2011) Oral tolerance. Immunol Rev 241, 241-259.

37. Hyytiainen M, Penttinen C \& Keski-Oja J (2004) Latent TGF-beta binding proteins: extracellular matrix association and roles in TGF-beta activation. Crit Rev Clin Lab Sci $\mathbf{4 1}$, $233-264$. 
38. Rubtsov YP \& Rudensky AY (2007) TGFbeta signalling in control of T-cell-mediated self-reactivity. Nat Rev Immunol 7, 443-453.

39. Kohm AP, Williams JS, Bickford AL, et al. (2005) Treatment with nonmitogenic anti-CD3 monoclonal antibody induces $\mathrm{CD}^{+} \mathrm{T}$ cell unresponsiveness and functional reversal of established experimental autoimmune encephalomyelitis. $J$ Immunol 174, 4525-4534.

40. Oida T \& Weiner HL (2011) Murine CD4 T cells produce a new form of TGF-beta as measured by a newly developed TGF-beta bioassay. PLoS One 6, e18365.

41. Li MO, Wan YY \& Flavell RA (2007) T cell-produced transforming growth factor-beta1 controls $\mathrm{T}$ cell tolerance and regulates Th1- and Th17-cell differentiation. Immunity 26, 579-591.

42. Nakamura K, Kitani A \& Strober W (2001) Cell contactdependent immunosuppression by $\mathrm{CD} 4(+) \mathrm{CD} 25(+)$ regulatory $\mathrm{T}$ cells is mediated by cell surface-bound transforming growth factor beta. J Exp Med 194, 629-644.

43. Oida T \& Weiner HL (2010) TGF-beta induces surface LAP expression on murine CD4 T cells independent of Foxp3 induction. PLoS One 5, e15523.

44. Miller A, Lider O \& Weiner HL (1991) Antigen-driven bystander suppression after oral administration of antigens. $J$ Exp Med 174, 791-798.

45. Zhang J, Markovic-Plese S, Lacet B, et al. (1994) Increased frequency of interleukin 2-responsive $\mathrm{T}$ cells specific for myelin basic protein and proteolipid protein in peripheral blood and cerebrospinal fluid of patients with multiple sclerosis. J Exp Med 179, 973-984.

46. Sun JB, Czerkinsky C \& Holmgren J (2010) Mucosally induced immunological tolerance, regulatory $\mathrm{T}$ cells and the adjuvant effect by cholera toxin B subunit. Scand J Immunol 71, 1-11.

47. Sun JB, Czerkinsky C \& Holmgren J (2012) B lymphocytes treated in vitro with antigen coupled to cholera toxin B subunit induce antigen-specific Foxp $3(+)$ regulatory T cells and protect against experimental autoimmune encephalomyelitis. J Immunol 188, 1686-1697.

48. George-Chandy A, Eriksson K, Lebens M, et al. (2001) Cholera toxin B subunit as a carrier molecule promotes antigen presentation and increases CD40 and CD86 expression on antigen-presenting cells. Infect Immun 69, 5716-5725.

49. Khoury SJ, Hancock WW \& Weiner HL (1992) Oral tolerance to myelin basic protein and natural recovery from experimental autoimmune encephalomyelitis are associated with downregulation of inflammatory cytokines and differential upregulation of transforming growth factor beta, interleukin 4, and prostaglandin E expression in the brain. J Exp Med 176, 1355-1364.

50. Slavin AJ, Maron R \& Weiner HL (2001) Mucosal administration of IL-10 enhances oral tolerance in autoimmune encephalomyelitis and diabetes. Int Immunol 13, 825-833.

51. Belghith M, Bluestone JA, Barriot S, et al. (2003) TGF-betadependent mechanisms mediate restoration of self-tolerance induced by antibodies to CD3 in overt autoimmune diabetes. Nat Med 9, 1202-1208.

52. Ochi H, Abraham M, Ishikawa H, et al. (2008) New immunosuppressive approaches: oral administration of CD3-specific antibody to treat autoimmunity. I Neurol Sci 274, 9-12.

53. Frenkel D, Huang Z, Maron R, et al. (2005) Neuroprotection by IL-10-producing MOG $\mathrm{CD}^{+}{ }^{+} \mathrm{T}$ cells following ischemic stroke. J Neurol Sci 233, 125-132. 